\title{
Leucemias crónicas y SARS-CoV-2
}

\section{Gilberto Barranco-Lampón ${ }^{1,2}$, Elena Tuna-Aguilar³ y Rafael Hurtado-Monroy**}

${ }^{1}$ Departamento de Hematología, Hospital General de México; ${ }^{2}$ Departamento de Hematología, Instituto Nacional de Cancerología; ${ }^{3}$ Departamento de Hematología, Instituto Nacional de Ciencias Médicas y Nutrición Salvador Zubirán; ${ }^{4}$ Departamento de Hematología, Hospital Ángeles del Pedregal. Ciudad de México, México

\section{Resumen}

El 30 de enero del año 2020, la Organización Mundial de la Salud declaró emergencia internacional de Salud Pública la pandemia causada por el coronavirus 2 del síndrome respiratorio agudo grave (SARS-CoV-2). Al inicio surgieron muchas dudas relacionadas con los ajustes al tratamiento y el seguimiento de los pacientes con leucemias crónicas y diversas asociaciones internacionales emitieron recomendaciones. El uso de la telemedicina y la selección de estrategias de tratamiento que permitan un menor acercamiento de los pacientes a los centros hospitalarios ha sido una de las principales técnicas de protección de los pacientes con leucemias crónicas. La experiencia internacional nos describe que al parecer los pacientes con leucemia mieloide crónica son menos susceptibles a contraer la infección y a morir, al contrario de los pacientes con leucemia linfocítica crónica.

PALABRAS CLAVE: Leucemia mieloide crónica. Leucemia linfocítica crónica. Coronavirus. SARS-CoV-2.

\section{Chronic leukemias and SARS-CoV-2}

\begin{abstract}
On January 30 of the 2020 year, the World Health Organization declared an international public health emergency the pandemic caused by the severe acute respiratory syndrome coronavirus 2 (SARS-CoV-2). Initially, many doubts arose regarding treatment adjustments and follow-up of patients with chronic leukemias, and various international associations issued recommendations. The use of telemedicine and the selection of treatment strategies that allow care without patients going to hospital centers, has been one of the main techniques for protecting patients with chronic leukemias. International experience describes that it seems that patients with chronic myeloid leukemia are less susceptible to infection and death, unlike patients with chronic lymphocytic leukemia.
\end{abstract}

KEY WORDS: Chronic myeloid leukemia. Chronic lymphocytic leukemia. Coronavirus. SARS-CoV-2.

\section{Introducción}

En enero 30 del año actual, la Organización Mundial de la Salud declaró emergencia internacional de salud pública la pandemia causada por el coronavirus 2 del síndrome respiratorio agudo grave (SARSCoV-2), cuya infección puede ser asintomática o un cuadro respiratorio grave, con gran impacto en el tejido hematopoyético y hemostásico que conduce a complicaciones cardiovasculares que pueden ser fatales.

La fisiopatología que se conoce implica que el SARS-CoV-2 reconoce al receptor de la enzima convertidora de angiotensina 2 (ACE2) por las proteínas de sus «picos». Este receptor se expresa en diversos
Correspondencia:

*Rafael Hurtado-Monroy

E-mail: rafahurtado@prodigy.net.mx
Fecha de recepción: 09-09-2020

Fecha de aceptación: 09-02-202

DOI: 10.24875/GMM.M21000469
Gac Med Mex. 2021;157(Supl3):S42-S46

Disponible en PubMed

www.gacetamedicademexico.com CC BY-NC-ND (http://creativecommons.org/licenses/by-nc-nd/4.0/). 
tejidos en los humanos, entre los que se incluyen: alveolos tipo 2, epitelio capilar, corazón, hígado, riñones y células endoteliales. La ACE2 se expresa también en células madre y precursoras hematopoyéticas, estimula su movilización y migración a las áreas de daño vascular y revascularización de las zonas de isquemia por condiciones de hipoxia.

Existe la probabilidad de que la vía de entrada de la célula huésped sea el receptor CD147, que se conoce también como BASIGI o EMMPRIN; a este receptor se le reconoce como el mismo en glóbulos rojos para el parásito Plasmodium falciparum y receptor vascular para Neisseria meningitidis. Este año Hoffman, et al. informaron que la serina proteasa celular TMPRSS2 para el «pico» del virus es la clave de entrada y su diseminación. Estos conocimientos podrían estimular nuevos agentes contra esta enfermedad ante la falta de un tratamiento específico'.

La pandemia de COVID-19 ha tenido impacto en el tratamiento de múltiples enfermedades y las leucemias crónicas no han sido excepción. Al inicio surgieron muchas dudas relacionadas con los ajustes al tratamiento y el seguimiento de los pacientes, y diversas asociaciones internacionales emitieron recomendaciones, las cuales resumiremos a continuación.

\section{Recomendaciones leucemia mieloide crónica}

Se resumen las recomendaciones emitidas por grupos de expertos ${ }^{2-4}$.

- Pacientes de reciente diagnóstico:

- No retrasar el inicio de tratamiento con inhibidores de la tirosina cinasa (TKI); extremar precauciones durante los primeros tres meses, riesgo de citopenias aumentado.

- Pacientes en tratamiento regular:

- Reducir al mínimo las visitas hospitalarias.

- Uso de la telemedicina.

- No interrumpir el tratamiento con TKI.

- Si el paciente presenta falla o intolerancia al tratamiento con alguno de los TKI, no retrasar el cambio a otro (en la medida de lo posible).

- Si el contexto de la pandemia lo permite, continuar con el monitoreo de los transcritos de $\mathrm{BCR} / \mathrm{ABL}$ mediante reacción en cadena de la polimerasa cuantitativa; en caso contrario, evaluar la toxicidad y respuesta mediante estudios de citometría hemática.

- No se recomienda iniciar intentos de descontinuación del tratamiento.
- Pacientes que desarrollan sintomatología compatible con COVID-19:

- En casos confirmados/sospechosos de COVID-19 con síntomas no severos, se recomienda continuar con el tratamiento con TKI.

- En casos confirmados/sospechosos de COVID-19 con síntomas severos, la decisión debe individualizarse teniendo en cuenta que todos los TKI tienen la capacidad de prolongar el intervalo QTc, en caso de requerir medicamentos que potencialmente favorezcan interacciones.

\section{Recomendaciones leucemia linfocítica crónica}

Se resumen las recomendaciones emitidas por grupos de expertos ${ }^{5,6}$.

- Pacientes en vigilancia:

- Reducir al mínimo las visitas hospitalarias.

- Uso de la telemedicina.

- Pacientes con indicación para iniciar tratamiento o que están activos con TKI:

- Posponer el inicio del tratamiento en la medida de lo posible.

- En aquellos pacientes que requieran iniciar tratamiento de inmediato, seleccionar el mejor tratamiento disponible de acuerdo con el perfil de cada paciente. En caso de contar con varias alternativas, dar preferencia a aquellas que puedan recibirse fuera del hospital.

- Evitar el uso de anticuerpos monoclonales y de tratamientos que ameriten controles de laboratorio frecuentes como el venetoclax.

- En pacientes que reciban terapias orales continuas no se recomienda interrumpir el tratamiento.

- Pacientes que desarrollen sintomatología compatible con COVID-19:

- En casos confirmados/sospechosos de COVID-19 con buen control de la leucemia linfocítica crónica (LLC) y con síntomas no severos, que se encuentren en tratamiento con inmunoquimioterapia, se recomienda suspender el tratamiento hasta que las condiciones de la pandemia lo permitan.

- En casos confirmados/sospechosos de COVID-19 con síntomas severos, se sugiere interrumpir el tratamiento con inmunoquimioterapia, inhibidores BCL2 y en el caso de los inhibidores de la tirosina cinasa de Bruton (BTK), individualizar la decisión por el riesgo 
Tabla 1. Reportes de casos publicados hasta el momento.

\begin{tabular}{|c|c|c|c|c|c|c|c|c|}
\hline Referencia & $n$ & Edad & $\begin{array}{l}\text { Fase al } \\
\text { Dx de } \\
\text { LMC }\end{array}$ & $\begin{array}{l}\text { TKI al Dx de } \\
\text { COVID-19 }\end{array}$ & $\begin{array}{l}\text { Respuesta } \\
\text { al Dx de } \\
\text { COVID-19 }\end{array}$ & Comorbilidad & $\begin{array}{l}\text { Tratamientos } \\
\text { acompañantes }\end{array}$ & VoM \\
\hline $\begin{array}{l}\text { Abruzzese, et al., } \\
2020^{12} \text { Italia }\end{array}$ & 1 & 29 & $\begin{array}{l}\text { FC } \\
\text { embarazo }\end{array}$ & $\begin{array}{l}\text { Dasatinib } \\
\text { No suspendió }\end{array}$ & RMM & No & Amoxicilina/Ac. clavulánico & V \\
\hline $\begin{array}{l}\text { Li, et al., } 2020^{13} \\
\text { China }(18 / 05 / 20)\end{array}$ & 5 & $\begin{array}{c}\text { Mediana } \\
47(41-89)\end{array}$ & $\begin{array}{l}F C: 3 \\
F A \text { o FB: } 2\end{array}$ & $\begin{array}{l}\text { Imatinib: } 3 \\
\text { No suspendieron } \\
\text { Ponatinib: } 1 \\
\text { Suspendió }\end{array}$ & $\begin{array}{l}\text { No RHC: } 2 \\
\text { RCyC: } 1 \\
\text { RMM: } 1\end{array}$ & $\begin{array}{l}\text { Cardiovascular: } 3 \\
\text { Diabetes: } 1\end{array}$ & & $\begin{array}{l}V: 5 \\
M: 0\end{array}$ \\
\hline $\begin{array}{l}\text { Abdalhad, et al., } \\
2020^{14} \text { Qatar } \\
(19 / 05 / 20)\end{array}$ & 1 & 65 & FA & $\begin{array}{l}\text { Dasatinib } \\
\text { Suspendió }\end{array}$ & RMM & No & $\begin{array}{l}\text { Hidroxicloroquina } \\
\text { Azitromicina } \\
\text { Oseltamivir } \\
\text { Piperacilina } \\
\text { Tazobactam } \\
\text { Ritonavir } \\
\text { Lopinavir } \\
\text { Tocilizumab } \\
\text { Metilprednisolona }\end{array}$ & V \\
\hline $\begin{array}{l}\text { Ranger, et al., } \\
2020^{15} \text { Reino } \\
\text { Unido }(01 / 06 / 20)\end{array}$ & 1 & 52 & FA & $\begin{array}{l}\text { Imatinib } \\
\text { Suspendió }\end{array}$ & No $\mathrm{RHC}$ & No & $\begin{array}{l}\text { Ritonavir } \\
\text { Lopinavir } \\
\text { Antibióticos amplio } \\
\text { espectro } \\
\text { Tocilizumab }\end{array}$ & V \\
\hline
\end{tabular}

Dx: diagnóstico; LMC: leucemia mieloide crónica; FC: fase crónica; FA: fase acelerada; FB: fase blástica; TKI: inhibidores de la tirosina cinasa; COVID-19: enfermedad por coronavirus 2019; RMM: respuesta molecular mayor; RHC: respuesta hematológica completa; RCyC: respuesta citogenética completa; V: vivo; M: muerto.

de interacciones con los fármacos utilizados para el tratamiento de la COVID-19. Vigilar estrechamente los datos actividad de la leucemia mientras el tratamiento permanece en suspensión.

\section{Coronavirus e inhibidores de la tirosina cinasa}

Se ha postulado que los TKI, particularmente el imatinib, pueden tener un papel en el tratamiento de los pacientes con infección por coronavirus, incluyendo el coronavirus del síndrome respiratorio agudo severo (SARS-CoV) y el coronavirus del síndrome respiratorio del Medio Oriente? ${ }^{7}$. En la leucemia mieloide crónica (LMC) se encontró que entre los pacientes en tratamiento con imatinib su frecuencia de infección por SARS-CoV-2 es menor que en otros padecimientos predisponentes y se postula que el imatinib puede tener un efecto protector en bloquear la fusión de la proteína $S$ de la superficie viral con las membranas celulares ${ }^{7,8}$.

Estas investigaciones, sumadas a la baja incidencia y letalidad reportada en los países que se encuentran en fases más avanzadas de la pandemia, sugieren que el imatinib pudiera utilizarse en el tratamiento de los pacientes con infección por SARS-CoV-2, independientemente de si padecen LMC o no, y se están llevando a cabo ensayos clínicos que respondan esta interrogante ${ }^{9}$.

\section{Inhibidores de la tirosina cinasa de Bruton y coronavirus}

Se ha postulado que el ibrutinib, y posiblemente otros inhibidores de la BTK, pueden proporcionar protección contra la lesión pulmonar e incluso mejorar la función pulmonar en pacientes hipóxicos con COVID-19 al disminuir las citocinas inflamatorias pulmonares y la lesión pulmonar, como se observó en modelos animales y en una serie de pacientes con macroglobulinemia de Waldenström en tratamiento con ibrutinib. Estos hallazgos deben considerarse generadores de hipótesis y servir de base para estudios más amplios ${ }^{10}$.

\section{Experiencia internacional en pacientes con leucemias crónicas y COVID-19}

Los miembros del grupo cooperativo italiano (The Campus CML) unieron sus experiencias en el tratamiento de la LMC y valoraron la incidencia de la COVID-19. Participaron 51 centros y 47 (92\%) 
completaron la encuesta en el periodo pandémico. De 6,883 pacientes con LMC solo 12 casos $(0.17 \%)$ se confirmaron a la mitad de abril del año actual y solo se reportaron dos muertes ${ }^{11}$.

En la tabla 1 se describen algunos reportes de casos publicados hasta el momento, el país de reporte, número de pacientes, fase de la LMC al diagnóstico de la enfermedad, el TKI utilizado y la respuesta en el momento del diagnóstico de COVID-19, si se suspendió o no el TKI, las comorbilidades, los tratamientos utilizados para la infección por COVID-19 y el desenlace.

La LLC es la leucemia con mayor prevalencia en los países occidentales. Estos pacientes suelen tener la inmunidad afectada, por lo tanto, era de esperarse una afectación especial a esta población. Los miembros del grupo cooperativo italiano unieron sus experiencias en el tratamiento de LLC durante el pico de la pandemia y valoraron la incidencia de la COVID-19. Participaron 33 centros, reuniendo 9,930 pacientes con LLC; 47 casos (0.5\%) se confirmaron a la mitad de abril del año actual y se obtuvo información de 46 . La infección se produjo tanto en pacientes tratados como no tratados. Más detalladamente, 16 pacientes con LLC nunca habían sido tratados, 15 estaban en tratamiento de primera línea o de rescate ( 5 con ibrutinib, 4 con quimioinmunoterapia, 6 con otros tratamientos), 9 estaban dentro de los seis meses posteriores a la interrupción de la quimioterapia y 6 habían sido tratados de manera temprana y estaban en vigilancia. Catorce pacientes nunca requirieron oxígeno/ventilación, mientras que 32 sí. A partir del 18 de mayo, 28 pacientes se han recuperado completamente, 4 están en el hospital, 2 con soporte de oxígeno no invasivo y 14 han muerto, con una tasa de mortalidad del $30.4 \%$ (mediana de edad: 75 ; rango: 51-91). A partir de la misma fecha (18 de mayo), la tasa de mortalidad para pacientes sintomáticos de COVID-19 entre la población general en Italia era del $13.4 \%$ y del $25.5 \%$ en la población de 70 a 79 años ${ }^{16}$.

\section{Conclusiones}

Los pacientes con leucemias crónicas son un grupo susceptible de contagio por SARS-CoV-2, por las características de su enfermedad y la necesidad de acudir a control periódico hospitalario. Al parecer, los pacientes con LMC son menos susceptibles a contraer la infección y a morir que los pacientes con LLC. El uso de la telemedicina ha sido una herramienta fundamental para el tratamiento y seguimiento de los pacientes con leucemias crónicas, pudiendo sustituir la visita hospitalaria en ciertas condiciones y disminuyendo de este modo el riesgo de contagio. Es probable que las estrategias de tratamiento que se han adaptado se fusionen con las establecidas en la época pre-COVID-19 para mejorar la calidad de vida y monitorización de los pacientes con estas enfermedades.

\section{Conflicto de intereses}

Los autores declaran no tener conflicto de intereses.

\section{Financiamiento}

La presente investigación no ha recibido ninguna beca específica de agencias de los sectores público, comercial, o sin ánimo de lucro.

\section{Responsabilidades éticas}

Protección de personas y animales. Los autores declaran que para esta investigación no se han realizado experimentos en seres humanos ni en animales.

Confidencialidad de los datos. Los autores declaran que en este artículo no aparecen datos de pacientes.

Derecho a la privacidad y consentimiento informado. Los autores declaran que en este artículo no aparecen datos de pacientes.

\section{Bibliografía}

1. Debuc B, Smadja DM. Is COVID-19 a New Hematologic Disease? Stem Cell Rev Reports [Internet]. 2020 May 12 [cited 2020 Jul 14];1-5. Available from: https://doi.org/10.1007/s12015-020-09987-4

2. Recommendations for specific hematologic malignancies [Internet]. [cited 2020 Jul 6]. Available from: https://ehaweb.org/covid-19/covid-19-recommendations/recommendations-for-specific-hematologic-malignancies/

3. COVID-19 and CML - Hematology.org [Internet]. [cited 2020 Jul 6]. Available from: https://www.hematology.org/covid-19/covid-19-and-cml

4. Recomendaciones sobre el manejo de pacientes con Leucemia Mieloide Crónica (LMC) en el seno de la pandemia por SARS-CoV-2/enfermedad COVID-19 [Internet]. [cited 2020 Jul 6]. Available from: https://www.sehh. es/covid-19/recomendaciones/123877-recomendaciones-del-gelmc-sobre-el-manejo-de-pacientes-con-leucemia-mieloide-cronica-Imc-en-el-seno-de-la-pandemia-por-sars-cov-2-enfermedad-covid-19

5. COVID-19 and CLL - Hematology.org [Internet]. [cited 2020 Jul 9]. Available from: https://www.hematology.org/covid-19/covid-19-and-cll

6. Guía para el manejo de pacientes con LLC e infección por SARS-CoV-2 [Internet]. [cited 2020 Jul 9]. Available from: https://www.sehh.es/covid-19/recomendaciones/123821-guia-para-el-manejo-de-pacientes-conIlc-e-infeccion-por-sars-cov-2

7. Sisk JM, Frieman MB, Machamer CE. Coronavirus S protein-induced fusion is blocked prior to hemifusion by Abl kinase inhibitors. J Gen Virol [Internet]. 2018 May 1 [cited 2020 Jun 29];99(5):619-30. Available from: http://www.ncbi.nlm.nih.gov/pubmed/29557770

8. Coleman CM, Sisk JM, Mingo RM, Nelson EA, White JM, Frieman MB. Abelson Kinase Inhibitors Are Potent Inhibitors of Severe Acute Respiratory Syndrome Coronavirus and Middle East Respiratory Syndrome Coronavirus Fusion. J Virol. 2016 Oct 1;90(19):8924-33.

9. Search of: imatinib | COVID - List Results - ClinicalTrials.gov [Internet]. [cited $2020 \mathrm{Jul} 6$ ]. Available from: https://clinicaltrials.gov/ct2/results?con$\mathrm{d}=$ COVID\&term $=$ imatinib\&cntry $=\&$ state $=\&$ city $=\&$ dist $=$ 
Gaceta Médica de México. 2021;157(Supl 3)

10. Treon SP, Castillo JJ, Skarbnik AP, Soumerai JD, Ghobrial IM, Guerrera ML, et al. The BTK inhibitor ibrutinib may protect against pulmonary injury in COVID-19-infected patients. Blood [Internet]. 2020 May 21 [cited 2020 Jul 9];135(21):1912-5. Available from: https://ashpublications.org/blood/article-pdf/135/21/1912/1731378/bloodbld2020006288.pdf

11. Breccia M, Abruzzese E, Bocchia M, Bonifacio M, Castagnetti F, Fava C et al. Chronic myeloid leukemia management at the time of the COVID-19 pandemic in Italy. A campus CML survey. Leukemia [Internet] 2020 Jun 18 [cited 2020 Jun 29]; Available from: http://www.ncbi.nlm.nih. gov/pubmed/32555369

12. Abruzzese E, Luciano L, D'Agostino F, Trawinska MM, Pane F, de Fabritiis P. SARS-CoV-2 (CoVID-19) and chronic myeloid leukemia (CML) $A$ case report and review of $\mathrm{Abl}$ kinase involvement in viral infection [nternet]. Vol. 12, Mediterranean Journal of Hematology and Infectious Diseases. Universita Cattolica del Sacro Cuore; 2020 [cited 2020 Jul 6]. p. 2020031. Available from: www.mjhid.org

13. Li W, Wang D, Guo J, Yuan G, Yang Z, Gale RP, et al. COVID-19 in persons with chronic myeloid leukaemia. Leukemia [Internet]. 2020 [cited 2020 Jun 29]; Available from: https://pubmed.ncbi.nlm.nih.gov/32424293/
14. Abdalhadi AM, Alshurafa A, Alkhatib M, Abou Kamar M, Yassin MA. Confirmed Coronavirus Disease-19 (COVID-19) in a Male with Chronic Myeloid Leukemia Complicated by Febrile Neutropenia and Acute Respiratory Distress Syndrome. Case Rep Oncol [Internet]. 2020 [cited 2020 Jun 29];13(2):569-77. Available from: http://www.ncbi.nlm.nih.gov/pubmed/32547383

15. Ranger A, Haji R, Kaczmarski R, Danga A. Interleukin 6 Blockade Treatment for COVID-19 associated Cytokine Release Syndrome in a Patient with Poorly Controlled Chronic Myeloid Leukaemia. Br J Haematol [Internet]. 2020 Jun [cited 2020 Jul 6]; Available from: https://pubmed.ncbi. nlm.nih.gov/32480422/

16. Cuneo A, Reda Fondazione IRCCS Cà Granda Ospedale Maggiore Policlinico G, Marzia Varettoni Fondazione IRCCS Policlinico San Matteo I, Francesca Maria Quaglia I, De Paoli Amedeo L, Matteo Rigolin G, et al. CHRONIC LYMPHOCYTIC LEUKEMIA MANAGEMENT IN ITALY DURING THE COVID-19 PANDEMIC. A CAMPUS CLL REPORT Clinical trial registration information (if any): CHRONIC LYMPHOCYTIC LEUKEMIA MANAGEMENT IN ITALY DURING THE COVID-19 PANDEMIC. A CAMPUS CLL REPORT. [cited $2020 \mathrm{Jul}$ 9]; Available from: https://ashpublications.org/blood/article-pdf/ doi/10.1182/blood.2020006854/1745561/blood.2020006854.pdf 